\title{
CIRCLE BUNDLES AND THE KRECK-STOLZ INVARIANT
}

\author{
XIANZHE DAI AND WEIPING ZHANG
}

\begin{abstract}
We present a direct analytic calculation of the $s$-invariant of KreckStolz for circle bundles, by evaluating the adiabatic limits of $\eta$ invariants. We believe that this method should have wider applications.
\end{abstract}

\section{INTRODUCTION}

Let $M$ be a $4 k-1$ dimensional closed spin manifold with vanishing real Pontrjagin classes and a metric of positive scalar curvature. In [KS] Kreck and Stolz introduced a very interesting invariant of such manifold. This so-called $s$-invariant is an absolute version of a relative invariant introduced by GromovLawson [GL], and plays a critical role in Kreck-Stolz's study of the moduli spaces of positive sectional curvature metrics.

In particular, a calculation of the $s$-invariant for circle bundles is very crucial for both of the main results in [KS]. This is achieved using cobordism theory in [KS]. In this note we present a direct analytic calculation by evaluating the adiabatic limits of $\eta$ invariants as well as the characteristic forms appearing in the definition of the $s$-invariant.

Acknowledgment. The first author would like to thank Stephan Stolz for interesting discussions.

\section{The $s$-INVARIANT OF KRECK-STOLZ}

Let $M$ be a closed $4 k-1$ dimensional spin manifold with vanishing real Pontrjagin classes. Let $g$ be a metric of positive scalar curvature on $M$. We recall the Q-valued invariant $s(M, g)$ introduced in [KS]. This invariant is related to an integer valued invariant $i\left(g_{1}, g_{2}\right)$ defined by Gromov and Lawson [GL] for a pair of positive scalar curvature metrics $g_{1}, g_{2}$ on $M$. More precisely,

$$
i\left(g_{1}, g_{2}\right)=s\left(M, g_{1}\right)-s\left(M, g_{2}\right) .
$$

Received by the editors December 4, 1994.

1991 Mathematics Subject Classification. Primary 58G10; Secondary 58G11, 53C20.

The first author was supported in part by NSF Grant \# DMS9204267 and Alfred P. Sloan Fellowship.

The second author was supported in part by the Chinese National Science Foundation. 
These invariants are closely related to the Dirac operator on manifolds with boundary and its index, which explains the integrality or rationality of these invariants.

Remark. The definition of Gromov-Lawson invariant does not require the vanishing of the real Pontryagin classes.

As in [KS], if $\alpha, \beta$ are two exact forms on $M$, then we define

$$
\int_{M} d^{-1}(\alpha \wedge \beta)=\int_{M} \hat{\alpha} \wedge \beta
$$

where $d \hat{\alpha}=\alpha$. Since $\beta$ is also exact one verifies easily that the definition does not depend on the choice of $\hat{\alpha}$.

Now if $W$ is a compact manifold with boundary $\partial W=M$, we have the long exact sequence for the de Rham cohomologies:

$$
\cdots \rightarrow H^{*}(W, \partial W) \stackrel{j}{\rightarrow} H^{*}(W) \rightarrow H^{*}(M) \rightarrow \cdots .
$$

Thus if $\alpha, \beta$ represent relative de Rham classes in $H^{*}(W, \partial W)$, then $\left.\alpha\right|_{\partial W}=$ $d \hat{\alpha}$ (and similarly for $\beta$ ). An immediate application of Stokes' Theorem yields

$$
\int_{M} d^{-1}(\alpha \wedge \beta)=\int_{W} \alpha \wedge \beta-\langle[\alpha] \cup[\beta],[W, \partial W]\rangle \text {. }
$$

Set

$$
a_{k}=\frac{1}{2^{2 k+1}\left(2^{2 k-1}-1\right)} .
$$

Denote by $B(M, g)$ (resp. $D(M, g))$ the signature (resp. Dirac) operator on $M$. We can now define the $s$-invariant [KS, Definition 2.12].

Definition. The $s$-invariant $s(M, g)$ is defined as

$$
s(M, g)=-\frac{1}{2} \eta(D(M, g))-a_{k} \eta(B(M, g))+\int_{M} d^{-1}\left(\hat{A}+a_{k} L\right)\left(p_{i}(M, g)\right),
$$

where $\eta(D(M, g))$ (resp. $\eta(B(M, g)))$ is the $\eta$-invariant of $D(M, g)$ (resp. $B(M, g))$ [APS], and $p_{i}(M, g)$ is the Pontrjagin form obtained via the ChernWeil theory for the Levi-Civita connection of $g$.

Remark. The choice of $a_{k}$ is precisely to cancel out the component in degree $4 k$ in $\left(\hat{A}+a_{k} L\right)\left(p_{i}(M, g)\right)$, leaving us with a linear combination of products of exact forms.

The usefulness of this invariant comes from the following basic properties of the invariant:

Proposition 2.1 (Kreck-Stolz). Let $M, M^{\prime}$ be closed $4 k-1$ dimensional spin manifolds with vanishing real Pontrjagin classes and let $g, g^{\prime}$ be positive scalar curvature metrics on $M, M^{\prime}$ respectively.

(a) If there exists an isometry between $(M, g)$ and $\left(M^{\prime}, g^{\prime}\right)$ preserving the spin structures, then $s(M, g)=s\left(M^{\prime}, g^{\prime}\right)$.

(b) $s(M, g)$ depends only on the connected component of $g$ in the space of metrics of positive scalar curvature on $M$. 
(c) If $M$ bounds a spin manifold $W$ with the metric $g_{W}$ extending $g$ and being the product metric near the boundary, then

$$
s(M, g)=\operatorname{ind} D^{+}\left(W, g_{W}\right)+t(W),
$$

where ind $D^{+}\left(W, g_{W}\right)$ denote the index of the Dirac operator on $W$ with the Atiyah-Patodi-Singer boundary condition [APS], and $t(W)$ is a topological invariant defined as

$$
t(W)=-\left\langle\left(\hat{A}+a_{k} L\right)\left(j^{-1} p_{i}(W)\right),[W, \partial W]\right\rangle+a_{k} \operatorname{sign}(W) .
$$

Here $j$ is the natural map $j: H^{*}(W, \partial W) \rightarrow H^{*}(W)$ from the long exact sequence.

(d) The s-invariant is additive under connected sum:

$$
s\left(M \# M^{\prime}, g \# g^{\prime}\right)=s(M, g)+s\left(M^{\prime}, g^{\prime}\right) .
$$

In the next section, we will give a direct computation of $s(M, g)$ where $M$ is a circle bundle and $g$ is $S^{1}$-equivariant.

\section{THE $S$-INVARIANT OF CIRCLE BUNDLES: A COMPUTATION VIA ADIABATIC LIMIT}

Let $B$ be a $4 k-2$ dimensional closed spin manifold and $g^{T B}$ a metric of positive scalar curvature on $B$. Let $\pi: N \rightarrow B$ be an oriented two dimensional real vector bundle over $B$ and $g^{N}$ a fiber metric on $N$ with $\nabla^{N}$ a compatible connection. Thus if we denote $R^{N}=\left(\nabla^{N}\right)^{2}$ the curvature and $T=\operatorname{Pf}\left(R^{N}\right)$ the Pfaffian, then $\frac{T}{2 \pi}$ represents the Euler class $e$ of $N$.

The connection $\nabla^{N}$ determines a horizontal subbundle $T^{H} N$ of $T N$. Let $g^{T N}=g^{N} \oplus \pi^{*}\left(g^{T B}\right)$. Let $M$ be the unit sphere bundle of $N$ with the induced metric $g^{T M}$. Then $M$ is a circle bundle over $B$ with the holonomy group $U(1)$ acting by isometries and carries an induced spin structure. (This is the spin structure $\phi$ if we adopt the notation of [KS].)

Since $g^{T B}$ has positive scalar curvature, a standard formula (cf. [KS, (4.4)]) shows that $g^{T M}$ also has positive scalar curvature (this may require shrinking the fiber metric; note that this is compatible with the rescaling in the adiabatic limit defined below). Assume now that all the real Pontrjagin classes of $M$ vanish. The following formula for the $s$-invariant of $M$ is the key for all the applications in [KS].

Theorem 3.1 (Kreck and Stolz). The s-invariant of $M$ is given in terms of the Euler class of $N$ and the characteristic classes of $B$ as follows.

$$
s\left(M, \phi, g^{T M}\right)=-\left\langle\hat{A}(T B) \frac{1}{2 \tanh \frac{e}{2}}+a_{k} L(T B) \frac{1}{\tanh e},[B]\right\rangle+a_{k} \operatorname{sign}\left(B_{e}\right),
$$

where $\operatorname{sign}\left(B_{e}\right)$ is the signature of the bilinear form

$$
\begin{gathered}
B_{e}: H^{2 k-2}(B) \otimes H^{2 k-2}(B) \rightarrow R, \\
B_{e}(x \otimes y)=\langle x y e,[B]\rangle .
\end{gathered}
$$

This is proved in [KS] by using indirect cobordism techniques. 
Since $s\left(M, \phi, g^{T M}\right)$ is defined in terms of intrinsic analytic invariants, it would be more natural and helpful to provide a direct geometric proof of Theorem 3.1. Using adiabatic limit we present such a proof.

For $\epsilon>0$, let

$$
g_{\epsilon}=g_{\epsilon}^{T M}=g^{N} \oplus \pi^{*}\left(\frac{1}{\epsilon} g^{T B}\right) .
$$

Clearly $\left(M, g_{\epsilon}\right)$ still satisfies the requirement in Definition 1.1, so the $s$-invariant $s\left(M, g_{\epsilon}\right)$ is still defined. Furthermore, $\left(M, g_{\epsilon}\right)$ represents a continuous family of metrics of positive scalar curvature. Hence, from (2.1) $s\left(M, g_{\epsilon}\right)$ does not depend on $\epsilon$. limit.

We now take $\epsilon \rightarrow 0$. This procedure is referred to as taking the adiabatic

Theorem 3.2. We have

$$
\lim _{\epsilon \rightarrow 0} \frac{1}{2} \eta\left(D\left(M, g_{\epsilon}^{T M}\right)\right)=-\left\langle\hat{A}(T B)\left(\frac{1}{e}-\frac{1}{2 \tanh \frac{e}{2}}\right),[B]\right\rangle,
$$

and

$$
\lim _{\epsilon \rightarrow 0} \eta\left(B\left(M, g_{\epsilon}^{T M}\right)\right)=\left\langle L(T B)\left(\frac{1}{\tanh e}-\frac{1}{e}\right),[B]\right\rangle-\operatorname{sign}\left(B_{e}\right) .
$$

Proof. The first result is proved in [Z2, Theorem 2.5], by using the results and methods of Bismut-Cheeger [BC1] and Dai [D1]. The minus sign appears because of the choice of orientation, compare [Z1, Theorem 1]. The other terms disappear because $g^{T B}$ is of positive scalar curvature. Dai [D2] had also independently computed the adiabatic limits of $\eta$-invariants of Dirac operators on circle bundles. The novelty of $[\mathrm{Z} 1, \mathrm{Z} 2]$ is that Zhang found an application of this result to the Rokhlin type congruences.

For the second formula, let $N_{1}=\left\{u \in N \mid\|u\|_{g^{N}} \leq 1\right\}$ be the disc bundle with fibre $D$ over $B$. Clearly $M=\partial N_{1}$. It is easy to construct a metric $g^{T D}$ on $T D$ such that for any $\epsilon>0, g_{\epsilon}^{T N_{1}}=g^{T D}+\pi^{*}\left(\frac{1}{\epsilon} g^{T B}\right)$ is a product near $\partial N_{1}=M$ and $\left.g_{\epsilon}^{T N_{1}}\right|_{T M}=g_{\epsilon}^{T M}$.

Applying the Atiyah-Patodi-Singer index theorem for manifolds with boundary [APS] yields, for any $\epsilon>0$,

$$
\operatorname{sign}\left(N_{1}\right)=\int_{N_{1}} L\left(P_{i}\left(N_{1}, g_{\epsilon}^{T N_{1}}\right)\right)-\eta\left(B\left(M, g_{\epsilon}^{T M}\right)\right)
$$

Or

$$
\lim _{\epsilon \rightarrow 0} \eta\left(B\left(M, g_{\epsilon}^{T M}\right)\right)=-\operatorname{sign}\left(N_{1}\right)+\lim _{\epsilon \rightarrow 0} \int_{N_{1}} L\left(P_{i}\left(N_{1}, g_{\epsilon}^{T N_{1}}\right)\right) .
$$

But (cf. [BC1])

$$
\lim _{\epsilon \rightarrow 0} L\left(P_{i}\left(N_{1}, g_{\epsilon}^{T N_{1}}\right)\right)=L\left(P_{i}\left(B, g^{T B}\right)\right) L\left(P_{i}\left(D, g^{T D}\right)\right) .
$$

Since $g^{T D}$ is a product metric near the boundary, its curvature vanishes near the boundary, and therefore, represents (up to a constant) the Thom class of the vector bundle. Using the Thom isomorphism theorem, a straightforward 
computation shows

$$
\lim _{\epsilon \rightarrow 0} \int_{N_{1}} L\left(P_{i}\left(N_{1}, g_{\epsilon}^{T N_{1}}\right)\right)=\left\langle L(T B)\left(\frac{1}{\tanh e}-\frac{1}{e}\right),[B]\right\rangle
$$

(compare [Z2, Lemma 3.5]). Also using the Thom isomorphism theorem we have $\operatorname{sign}\left(N_{1}\right)=\operatorname{sign}\left(B_{e}\right)$, proving (3.3).

Proof of Theorem 3.1. For this purpose it suffices to compute the last term in Definition 1.1 , that is

$$
\lim _{\epsilon \rightarrow 0} \int_{M} d^{-1}\left(\hat{A}+a_{k} L\right)\left(p_{i}\left(M, g_{\epsilon}^{T M}\right)\right)
$$

Formula (2.4) gives

$$
\begin{aligned}
& \int_{M} d^{-1}\left(\hat{A}+a_{k} L\right)\left(p_{i}\left(M, g_{\epsilon}^{T M}\right)\right) \\
& \quad=\int_{N_{1}}\left(\hat{A}+a_{k} L\right)\left(p_{i}\left(N_{1}, g_{\epsilon}^{T N_{1}}\right)\right)-\left\langle\left(\hat{A}+a_{k} L\right)\left(p_{i}\left(N_{1}\right)\right),\left[N_{1}, M\right]\right\rangle .
\end{aligned}
$$

Proceeding as above we have

$$
\begin{aligned}
\lim _{\epsilon \rightarrow 0} \int_{N_{1}}(\hat{A} & \left.+a_{k} L\right)\left(p_{i}\left(N_{1}, g_{\epsilon}^{T N_{1}}\right)\right) \\
& =\left\langle\hat{A}(T B)\left(\frac{1}{2 \sinh \frac{e}{2}}-\frac{1}{e}\right)+a_{k} L(T B)\left(\frac{1}{\tanh e}-\frac{1}{e}\right),[B]\right\rangle .
\end{aligned}
$$

The second term in the right-hand side of (3.5) can be evaluated as in [KS, p. 840], using the bundle splitting $T N_{1}=\pi^{*}(T B) \oplus T D$ and the Thom isomorphism theorem

$$
\left\langle j^{-1}\left(\hat{A}+a_{k} L\right)\left(p_{i}\left(N_{1}\right)\right),\left[N_{1}, M\right]\right\rangle=\left\langle\hat{A}(T B) \frac{1}{2 \sinh \frac{e}{2}}+a_{k} L(T B) \frac{1}{\tanh e},[B]\right\rangle .
$$

Combining (3.2), (3.3), and (3.5)-(3.7), we have

$$
\begin{array}{r}
\lim _{\epsilon \rightarrow 0}\left[\frac{1}{2} \eta\left(D\left(M, g_{\epsilon}^{T M}\right)\right)+a_{k} \eta\left(B\left(M, g_{\epsilon}^{T M}\right)\right)-\int_{M} d^{-1}\left(\hat{A}+a_{k} L\right)\left(p_{i}\left(M, g_{\epsilon}^{T M}\right)\right)\right] \\
=\left\langle\hat{A}(T B) \frac{1}{2 \tanh \frac{e}{2}}+a_{k} L(T B) \frac{1}{\tanh e},[B]\right\rangle-a_{k} \operatorname{sign}\left(B_{e}\right) .
\end{array}
$$

This completes the proof of Theorem 3.1.

\section{REMARKS}

There is extensive work on the adiabatic limit of eta invariant (and other geometric invariants). In general if $M$ is an oriented manifold that has a 
fibration structure

$$
Y \rightarrow M \stackrel{\pi}{\rightarrow} B
$$

and $g_{M}$ a submersion metric,

$$
g_{M}=\pi^{*} g_{B}+g_{Y},
$$

then blowing up the metric in the horizontal direction by a factor $x^{-2}$ gives us a family of metrics $g_{x}$,

$$
g_{x}=x^{-2} \pi^{*} g_{B}+g_{Y} .
$$

A general formula for $\lim _{x \rightarrow 0} \eta\left(B\left(M, g_{x}\right)\right)$ is given in [D1], which, in fact, comes from a more general formula for Dirac operators (cf. [D1]), namely,

$$
\lim _{x \rightarrow 0} \eta\left(A_{x}\right)=2 \int_{B} \mathscr{L}\left(\frac{R^{B}}{2 \pi}\right) \wedge \tilde{\eta}+\eta\left(A_{B} \otimes \operatorname{ker} A_{Y}\right)+2 \tau,
$$

where $\tilde{\eta}$ is the the $\tilde{\eta}$-form of Bismut-Cheeger [BC1], $R^{B}$ is the curvature tensor of $g_{B}$ and $A_{B}$ denotes the signature operator on $B$ and $A_{Y}$ the family of signature operators along $\mathrm{Y}$. The integer $\tau$ is a topological invariant computable from the Leray spectral sequence.

More specifically, let $\left(E_{r}, d_{r}\right) \quad(r \geq 2)$ be the $E_{r}$-term of the Leray spectral sequence of the fibration $Y \rightarrow M^{n} \rightarrow B$. The orientation gives a natural basis $\xi_{2}$ on $E_{2}^{n}$ which then induces a basis $\xi_{r}$ on $E_{r}^{n}$ for each $r>2$. Consider the pairing

$$
\langle,\rangle_{r}: E_{r}^{p} \otimes E_{r}^{q} \longrightarrow \mathbf{R}, \quad \varphi \otimes \psi \longrightarrow\left(\varphi \cdot d_{r} \psi, \xi_{r}\right) .
$$

If $n=4 k-1$ (otherwise we set $\tau=0$ ) it can be verified that $\langle,\rangle_{r}$ is symmetric when restricted to $E_{r}^{2 k-1}$. Therefore it gives rise to a symmetric matrix whose signature we will denote by $\tau_{r}$. Define $\tau=\sum_{r \geq 2} \tau_{r}$.

In the case of circle bundles the terms on the right-hand side of (4.2) can be computed explicitly. For example

$$
\tilde{\eta}=2\left(\frac{1}{2 \tanh \frac{e}{2}}-\frac{1}{e}\right)
$$

and

$$
\tau=\operatorname{sign}\left(B_{e}\right) .
$$

Taking into account of the definition of $\mathscr{L}$ we obtain the same formula as (3.3)

There are other cases where these invariants are quite computable, for example [BC2]. We believe that the method we present above should have wider applications.

\section{REFERENCES}

[APS] M. F. Atiyah, V. K. Patodi, and I. M. Singer, Spectral asymmetry and Riemannian geometry. I. Math. Proc. Cambridge Philos. Soc. 77 (1975), 43-69

[BC1] J. -M. Bismut and J. Cheeger, $\eta$-invariants and their adiabatic limits, J. Amer. Math. Soc. 2 (1989), 33-70

[BC2] - Transgressed Euler classes of $S L(2 n, Z)$ vector bundles, adiabatic limits of eta invariants and special values of L-functions, Ann. Sci. École Norm. Sup. 25 (1992), 335391. 
[D1] X. Dai, Adiabatic limits, the non-multiplicativity of signature and Leray spectral sequence, J. Amer. Math. Soc. 4 (1991), 256-321.

[D2] , unpublished.

[GL] M. Gormov and H. B. Lawson, Positive scalar curvature and the Dirac operator on complete Riemannian manifolds, Publ. IHES, no. 58 (1983), 83-196.

[KS] M. Kreck and S. Stolz, Nonconnected moduli spaces of positive sectional curvature metrics, J. Amer. Math. Soc. 6 (1993), 825-850.

[Z1] W. Zhang, Eta invariants and Rokhlin congruences, C.R. Acad. Sci. Paris Ser. I 315(1992), 305-308.

[Z2] - Circle bundles, adiabatic limits of $\eta$-invariants and Rokhlin congruences, Ann. Inst. Fourier (Grenoble) 44 (1994), 249-270.

Department of Mathematics, University of Southern California, los Angles, CaliFORNIA 90089

E-mail address: xdai@math.usc.edu

Nankai Institute of Mathematics, Tianjin, 300071, P.R. China 